\title{
Commentary on Recent Development of Biological Computer
}

\section{Chun-Liang Lin ${ }^{*}$ and Wei-Xian Li}

Department of Electrical Engineering, National Chung Hsing University, Taichung 402, Taiwan

*Corresponding author: Chun-Liang Lin, Department of Electrical Engineering, National Chung Hsing University, Taichung 402, Taiwan, Tel: +886987051622; E-mail: chunlin@dragon.nchu.edu.tw

Received date: February 15, 2018; Accepted date: March 21, 2018; Published date: March 26, 2018

Copyright: $\odot 2018$ Lin CL, et al. This is an open-access article distributed under the terms of the Creative Commons Attribution License, which permits unrestricted use, distribution, and reproduction in any medium, provided the original author and source are credited.

\section{Commentary}

Biological computers, or, in short, biocomputers, use systems of biologically derived molecules, usually referred to DNA and proteins, to conduct computational calculation such as data storing, retrieving, and processing [1]. In addition to be used as a carrier of genetic information, DNA molecules are suitable for performing molecular calculations as well. The sequence within a DNA molecule allows researchers to program desired reactions and implement circuits with which other DNA molecules it can react. For example, removing a gene, opening a gene, or transferring certain genes to form other species, changing the traditional operation, thereby affect cell expression. It works like electronic engineers to design an electrical circuit by picking up and composing standard components which correspond the specifications and connect those components to manipulate the behavior of the cell for specific function in a quick and reliable way.

Structurally, a biocomputer involves a pathway or a series of metabolic pathways constituted by biological materials that are engineered to behave in a certain function when excited by the input. In the past decade, the development of biocomputers has been made possible by the expanding science of biologically based materials. From an engineering viewpoint, it can be viewed as the design and engineering of proteins with assembled structures.

In 2000, Gardner, Cantor and Collins combined two genes, those are interfered with each other, to realize a gene switch which could switch between two steady states so as to realize one-bit data storage [2]. At the same issue of the journal, Elowitz and Leibler implemented a simple biological oscillator with Escherichia coli; the modified Escherichia coli can inhibit each other and produce the cycle of oscillation, thereby controlling the fluorescent protein open and close so that the fluorescent protein can shine like a light bulb [3]. The two iconic papers subvert the traditional concept of biology and set an important milestone in synthetic biology.

Nowday, scientists use bacterial and DNA molecules to design the essential Boolean operations of gene elements that can perform digital logic operation, including the biological gates XOR, NAND, OR, NOT, AND, etc. [4-6]. Scientists used a modified DNA molecule to alter Escherichia coli. When stimulated with chemicals, the modified Escherichia coli has "switches" that resembles electrical equivalents of biological counterparts. Scientists have also demonstrated that implanting a single logic gate into bacterial cells and programming the cells allows them to be linked together by chemical signals to form more complex parts like those of the electronic components made today.

In 2016, Nielsen, Der, Shin, et al. published a paper introducing computer-aided biological circuit design [7] in which they constructed a design environment named Cello (an acronym for cellular logic).
Cello was developed based on Verilog, a hardware description language, to construct a user friendly environment; it was established to accelerate development of the genetic circuitry that allows Verilog to be converted into the linear DNA sequences so that they can be built and operated in living cells. The current version of Cello can construct one to three different inputs into a genetic circuit. Cello has also built a library accommodating a variety of biological logic gates with different repressors and insulators.

Professor Richard Kitney said "Logic gates are the fundamental building blocks in silicon circuitry that our entire digital age is based on. Without them, we could not process digital information" [8]. Recent research tasks have demonstrated that scientists can replicate these parts using bacteria and DNA, it is thus expected that these works could lead to a new generation of biological processors, whose applications in the information or numerical data processing could be as important as their electronic equivalents.

In the large-scale digital electronic circuits, logic gates are the fundamental components. A silicon logic gate uses normalized signal levels in DC voltage to represent logic 1 and logic 0 . Digital integrated circuit, aviation equipment, electronic communication, or computer motherboards are assembled by a large amount of silicon logic gates. Unfortunately, biological circuits and electronic circuits are vastly different in many ways. Structurally, genetic circuits are not as simple as electronic circuits. In the latter, components' characteristics, in general, do not change over time or worked under different environments but it would be changed in the genetic circuits. The input and output physical quantities of the electronic circuits are mostly the same, but they are not for the genetic circuits.

In recent years, the research team led by Lin was devoted to realize a class of biological gates and plenty of functional circuits from the viewpoint of electrical engineering [6,9]. The systematic study starts from realization of fundamental Boolean biological gates and clocks using intelligent computational approaches. A real structured genetic algorithm (RSGA) [10] which combines advantages of the traditional real genetic algorithm (RGA) with those of the structured genetic algorithm (SGA) was adopted as the design tool and applied as an optimization strategy for optimal model parameter and structure determination. For the genetic oscillator design, their proposed approach fulfills all types of genes by minimizing the order of oscillator while searching for the optimal network parameters. The design approach is shown to be capable of yielding genetic oscillators with the simplest structure.

The computational method has also been applied to the biological logic gate design [6]. For a nonlinear model with the cis-regular input function (CRIF), a multi-objective performance index was established which is closely related to the response accuracy of the logic state and gap between high and low logic levels. For the genetic oscillator design, 
it was shown that the approach performs better than traditional GAs in the sense of computational efficiency and performance.

The genetic clock lies in its role of triggering logic reaction for sequential biological circuits. Because biochemical reaction of the biological system is extremely slow, however, transition between minimal and maximal levels is relatively instantaneous for an ideal clock signal; it is thus not directly realizable in biological sense. That meant that it would be hard to directly synthesize a square wave generator as a genetic clock. However, Fourier series can be used to approximately realize a square wave as a finite summation of sinusoidal waves generated by genetic oscillators with different harmonic oscillating frequencies, in which the amplitude alternates at a steady frequency between the fixed minimal and maximal levels with the same duration [11]. Another type of genetic clock generator on the basis of a genetic oscillator and a toggle switch has also been developed [12]. In which, a general sine wave generator was used as an oscillator. A dual repressor was then used to connect two fundamental biologic circuits to trim the sine wave.

DNA possesses huge message storage capacity. Biocomputers enables DNA to hold the complex blueprints of living organisms. Actually, the storage capacity of a single gram of DNA can hold as much information as one trillion compact discs [13]. Erlich and Zielinski stated that the theoretical information storage capacity of DNA is up to 680 petabytes per gram [14]. Scientists could even recover and read DNA sequences from fossils. That meant that DNA as a storage medium could last thousands of years. Biological memory serves as one of the core parts of the synthetic biological computer that are used to memorize data, temporarily or eternally. Today, a fundamental silicon computer memory (unit) is mostly formed by transistors and circuit components. In the biological system, however, it is not directly applicable using the same idea for realization of memory module. Hu et al. have proposed design of two type of genetic random access memories, i.e. genetic static random access memory (GSRAM), and genetic dynamic random access memory (GDRAM) which were inspired by the traditional silicon SRAM and DRAM $[15,16]$.

Recently, research theme has also been moving forward by combining biological logic gates, genetic clocks, arithmetic logic unit (ALU) and control unit (CU) to form a fundamental bio central processing unit (CPU). Lin et al. present a genetic ALU design based on several genetic logic gates with different functions [17]. The system level's architecture consists of three parts: temp register, ALU and accumulator register. Using the concept of the ALU on the digital computer, fundamental functions of the genetic ALU realized by a full adder register have been realized.

A computer cannot work completely without the capability of conducting a sequence of logical or mathematical operations. To fulfill the function, there should be a $\mathrm{CU}$ inside the CPU to arrange instructions in order. The $\mathrm{CU}$ is a component of a computer's CPU that directs the operations of the processor in sequence. In the biological sense, Lin et al. have constructed a bio-CU as the command centre to coordinate a series of operational steps corresponding to instructions decoded from the bio-instructions to realize the fundamental function of the prototype biocomputer [18]. Inside the CU system there are ring counter, ALU and instruction decoder which can be realized using the previously developed gene logic circuits from the data base already built up. A fully functional biocomputer resulted after combination of memory, clock, ALU, CU and necessary input/out ports.
To date, biomedical applications of DNA computers have been widely attempted. For example, Diseases such as leukemia, it usually indiscriminately attacks healthy and unhealthy cells; using certain DNA-based gates can discriminate between healthy and unhealthy cells by sensing the different characteristics of the cells to protect the patient's immune system [19], by measuring the concentration of specific antibodies it is possible to determine whether one is suffering from certain particular disease. The research team led by Professor Merkx have succeeded in linking the presence of antibodies to a DNA computer [20]. As for drug delivery, the method proposed by Dr. Engelen has translated the presence of each antibody into a unique piece of DNA whereby the DNA computer can decide on the basis of the presence of one or more antibodies whether drug delivery is needed [20]. For medication, a potential application is to measure the quantity of therapeutic antibodies in the blood so that doctor can decide whether it is necessary to conduct extra medication. Cancer has been a major cause of human death and is the second leading cause of death globally [21]. The DNA computer possesses the potential for use in cancer detection and medication. Recently, Nature published a paper regarding DNA nanorobot, the research team developed a DNA nanorobot that can cut off blood supply to the tumor cells causing tumor necrosis and inhibiting tumor growth [22]. Microsoft has expressed its interests in the DNA computer for cancer treatment and organized a research team to deal with the issue. Their final goal is to create a microcomputer from DNA that can survive in cells and monitor for faults in bodily networks, once the diseased cancer cells are detected, it will be reprogrammed to restore it to the healthy state [23]. There are increasing applications which may revolutionize technology in other fields such as life sciences which are currently under development.

Currently, biological computing is already available which attempts to extract computing power from the collective action of large numbers of biological molecules. However, biological CPU being totally replaced by biological molecules remains in the far future. Undoubtedly, the invention of biological computers will bring an immense influence on the human civilization like that of the silicon computer. It would bring tremendous changes to the world and stimulate wide applications. The goals of research of systems biology and proteomics are long-term and undergoing. It can be expected that the impact resulted from biocomputer would be gradually increasing, collaboration and cross-disciplinary approaches to sciences is a must.

\section{Acknowledgements}

This research was sponsored in part by National Science Council, Taiwan under the Grants NSC-102-2218-E-005-012 and NSC-101-2221-E-005-015-MY3.

\section{References}

1. https://en.wikipedia.org/wiki/Biological_computing.

2. Gardner TS, Cantor CR, Collins JJ (2000) Construction of a genetic toggle switch in Escherichia coli. Nature 403: 339-342.

3. Elowitz MB, Leibler S (2000) A synthetic oscillatory network of transcriptional regulators. Nature 403: 335-338.

4. Wang B, Kitney RI, Joly N, Buck M (2011) Engineering modular and orthogonal genetic logic gates for robust digital-like synthetic biology. Nat Commun 2: 508.

5. Bonnet J, Yin P, Ortiz ME, Subsoontorn P, Endy D (2013) Amplifying genetic logic gates. Science 340: 599-603. 
Citation: Lin CL, Li WX (2018) Commentary on Recent Development of Biological Computer. J Phylogenetics Evol Biol 6: 195. doi: $10.4172 / 2329-9002.1000195$

Page 3 of 3

6. Chuang CH, Lin CL, Chang YC, Jennawasin T, Chen PK (2013) Design of synthetic biological logic circuits based on evolutionary algorithm. IET Syst Biol 7: 89-105.

7. Nielsen AA, Der BS, Shin J, Vaidyanathan P, Paralanov V, et al. (2016) Genetic circuit design automation. Science 352: 7341.

8. Imperial College London (2011) Scientists create computing building blocks from bacteria and DNA.

9. Chuang CH, Lin CL (2014) A novel synthesizing genetic logic circuit: frequency multiplier. IEEE/ACM Trans on Comput Biol Bioinform 11: 702-713.

10. Tsai CW, Lin CL, Huang CH (2011) Microbrushless DC motor control design based on real coded structural genetic algorithm. IEEE/ASME Trans Mechatronics 16: 151-159.

11. Lin CL, Chen PK (2014) Synthesizing periodic triggering signals with genetic oscillators. IET Syst Biol 8: 1-12.

12. Lin CL, Chen PK, Cheng YY (2015) Synthesising gene clock with toggle switch and oscillator. IET Syst Biol 9: 88-94.

13. Future For All (2018) Biological Computers.

14. Erlich Y, Zielinsk D (2017) DNA Fountain enables a robust and efficient storage architecture. Science 355: 950-954.

15. Hu YJ, Lin CL, Li WX (2017) Design of dynamic genetic memory. IET Syst Biol 11: 182-189.
16. Hu YJ, Lin CL, Li WX (2018) Circuit design for static genetic memory. IET J Engineering 22-28.

17. Lin CL, Kuo TY, Charoenkit N, Chen YY, Preuksakarn C (2017) Toward theoretical synthesis of biocomputer. IET Syst Biol 11:36-43.

18. Lin CL, Kuo TY, Li WX (2017) Synthesis of control unit for future biocomputer. INFORMS J Comput, under review, 2018.

19. Song T, Garg S, Mokhtar R, Bui H, Reif J (2016) Analog computation by DNA strand displacement circuits. ACS Synth Biol 5: 898-912.

20. Technology University (2017) DNA computer brings intellengent drugs'a step closer.

21. Forouzanfar MH, Afshin A, Alexander LT, Anderson HR, Bhutta ZA, et al. (2016) Global, regional, and national comparative risk assessment of 79 behavioural, environmental and occupational, and metabolic risks or clusters of risks, 1990-2015: a systematic analysis for the Global Burden of Disease Study 2015. Lancet 388: 1659-1724.

22. Li S, Jiang Q, Liu S, Zhang Y, Tian Y, et al. (2018) A DNA nanorobot functions as a cancer therapeutic in response to a molecular trigger invivo. Nat Biotechnol 36: 258-264.

23. Microsoft Story Labs (2018) How Microsoft computer scientists and researchers are working to 'solve' cancer. 